\title{
What Can We Learn from (Pseudo)Rapidity Distribution in High Energy Collisions?
}

\author{
Fu-Hu Liu, ${ }^{1}$ Tian Tian, ${ }^{1}$ Jian-Xin Sun, ${ }^{1,2}$ and Bao-Chun $\mathrm{Li}^{1}$ \\ ${ }^{1}$ Institute of Theoretical Physics, Shanxi University, Taiyuan, Shanxi 030006, China \\ ${ }^{2}$ School of Physics and Electronic Science, Shanxi Datong University, Datong, Shanxi 037009, China \\ Correspondence should be addressed to Fu-Hu Liu; fuhuliu@163.com
}

Received 1 November 2013; Accepted 20 February 2014; Published 8 April 2014

Academic Editor: Dugan O’Neil

Copyright (C) $2014 \mathrm{Fu}-\mathrm{Hu}$ Liu et al. This is an open access article distributed under the Creative Commons Attribution License, which permits unrestricted use, distribution, and reproduction in any medium, provided the original work is properly cited. The publication of this article was funded by SCOAP ${ }^{3}$.

Based on the (pseudo)rapidity distribution of final-state particles produced in proton-proton ( $p p$ ) collisions at high energy, the probability distributions of momenta, longitudinal momenta, transverse momenta (transverse masses), energies, velocities, longitudinal velocities, transverse velocities, and emission angles of the considered particles are obtained in the framework of a multisource thermal model. The number density distributions of particles in coordinate and momentum spaces and related transverse planes, the particle dispersion plots in longitudinal and transverse coordinate spaces, and the particle dispersion plots in transverse momentum plane at the stage of freeze out in high energy pp collisions are also obtained.

\section{Introduction}

Due to the complexity of high energy collisions, it is impossible to measure all quantities and characteristics. Instead, some quantities and characteristics can be measured by experiment [1], while others can be obtained from the theoretical explanations based on the experimental results.

In experiments, high energy proton-proton $(p p)$, protonnucleus $(p A)$, deuteron-nucleus $(d A)$, and nucleus-nucleus $(A A)$ collisions have been performed at available accelerators such as the Super Proton Synchrotron (SPS) [2-4] and colliders such as the Relativistic Heavy Ion Collider (RHIC) [5-8] and the Large Hadron Collider (LHC) [9-12]. Some quantities and characteristics such as the pseudorapidity (or rapidity) distributions, transverse momentum distributions, azimuthal correlations, flow effects, and some other interesting information have been obtained [13].

A lot of models were introduced in the past several decades. Different phenomenological mechanisms including initial interactions, intermediate processes, and final-state statistical laws have been proposed [14-19]. In a workshop held a few years ago at the CERN Theory Institute [20], many models reported their predictions for the heavy ion program at the LHC based on the explanations of the experimental results at the RHIC. Recently, some of the models are tested by $p p, p A$, and $A A$ collisions at the LHC. Meanwhile, these models are further tested in $p p, p A, d A$, and $A A$ collisions at the RHIC and SPS.

In the past years, we suggested a multisource thermal model [21-23] to describe the (pseudo)rapidity distributions, multiplicity distributions, transverse momentum distributions, azimuthal distributions, flow effects, and so forth. Some interesting quantities such as the temperature, speed of sound, and number of sources are obtained. It is noticed that some quantities and characteristics are related to others. Usually, based on a few distributions, other distributions can be obtained due to some modelling assumptions.

Very recently, the NA61/SHINE Collaboration reported the negatively charged pion productions in inelastic $p p$ collisions at $20-158 \mathrm{GeV} / \mathrm{c}$ at the SPS [1]. The rapidity spectrums are parameterized by the sum of two Gaussian functions symmetrically displaced with respect to midrapidity. It is interesting for us to give a further test for the multisource thermal model by using the rapidity spectrums in $p p$ collisions at SPS momenta (energies). According to the rapidity spectrums, we hope some other distributions can be obtained by the model. 


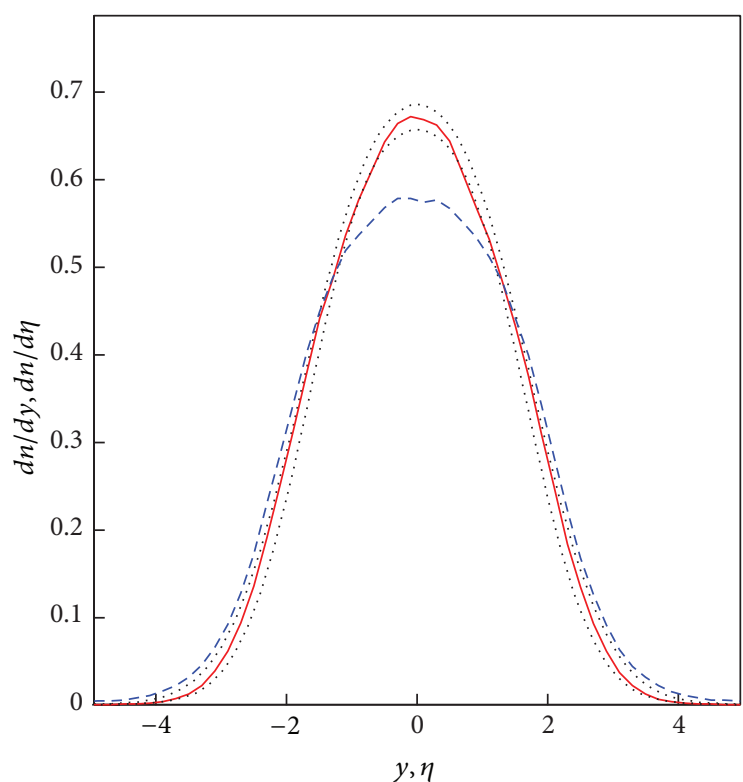

(a) $158 \mathrm{GeV} / \mathrm{c} p p, \pi^{-}$

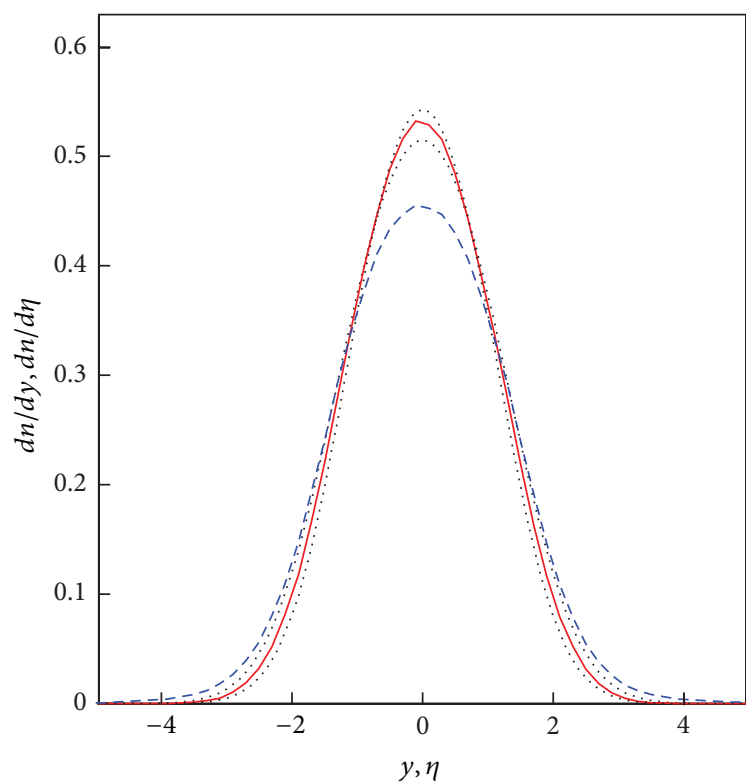

(c) $40 \mathrm{GeV} / \mathrm{c} p p, \pi^{-}$

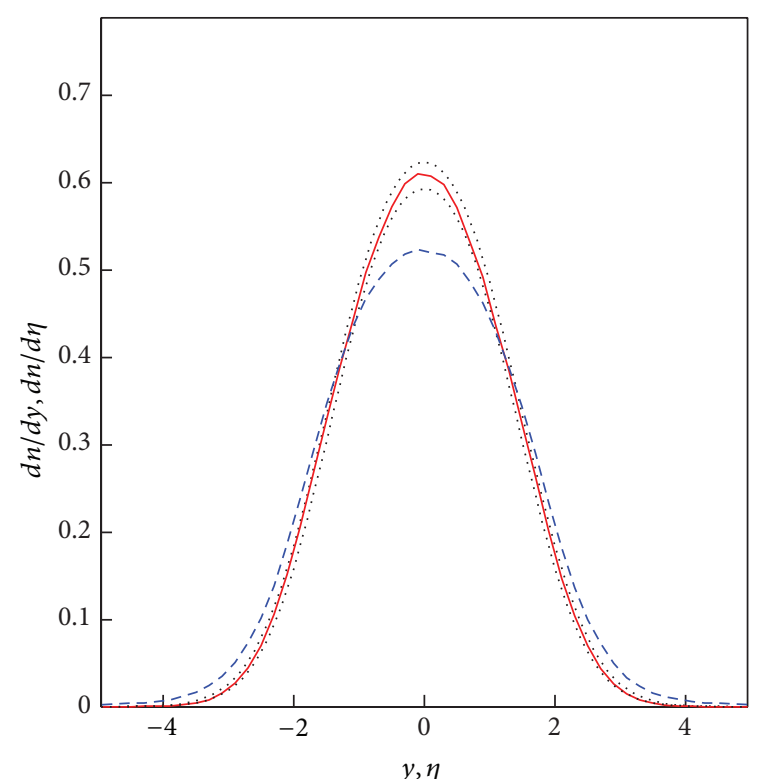

(b) $80 \mathrm{GeV} / \mathrm{c} p p, \pi^{-}$

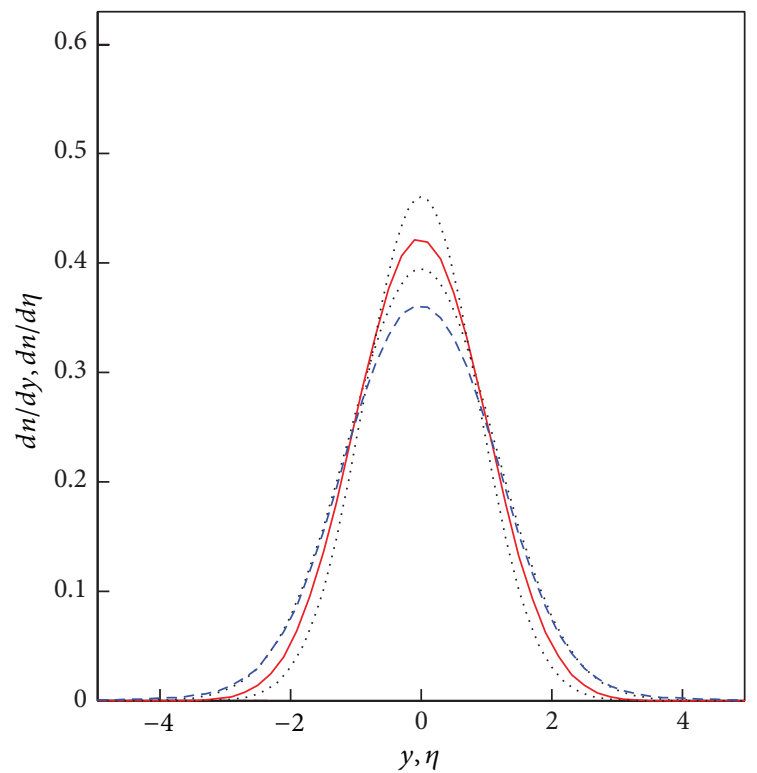

(d) $20 \mathrm{GeV} / \mathrm{c} p p, \pi^{-}$

FIGURE 1: Rapidity and pseudorapidity distributions of negatively charged pions produced in (a) 158, (b) 80 , (c) 40, and (d) $20 \mathrm{GeV} / \mathrm{c} p p$ collisions. The two-dotted curves represent the rapidity distribution range of the NA61/SHINE experiment [1]. The solid and dashed curves are our modelling results on the rapidity and pseudorapidity distributions.

In this paper, in the framework of the multisource thermal model [21-23], we analyze the rapidity spectrums in $p p$ collisions measured by the NA61/SHINE Collaboration at the SPS [1]. Then, a series of other distributions are obtained due to the description of rapidity spectrums.

\section{The Model and Calculation Method}

In the multisource thermal model [21-23], we assume that many emission sources are formed in high energy collisions. In rapidity space in the laboratory or centerof-mass reference frame, these sources distribute at different rapidity $y_{x}$ and form a target cylinder in rapidity interval $\left[y_{T \min }, y_{T \max }\right]$ and a projectile cylinder in rapidity interval $\left[y_{P \min }, y_{P \max }\right]$, respectively. Because of the symmetry in $p p$ collision, we have $\left[y_{T \min }=-y_{P \max }\right]$ and $\left[y_{T \max }=-y_{P \min }\right]$.

In the rest frame of a given source, we can use different formalizations to describe the production of particles. For example, we can use the relativistic ideal gas model to give 


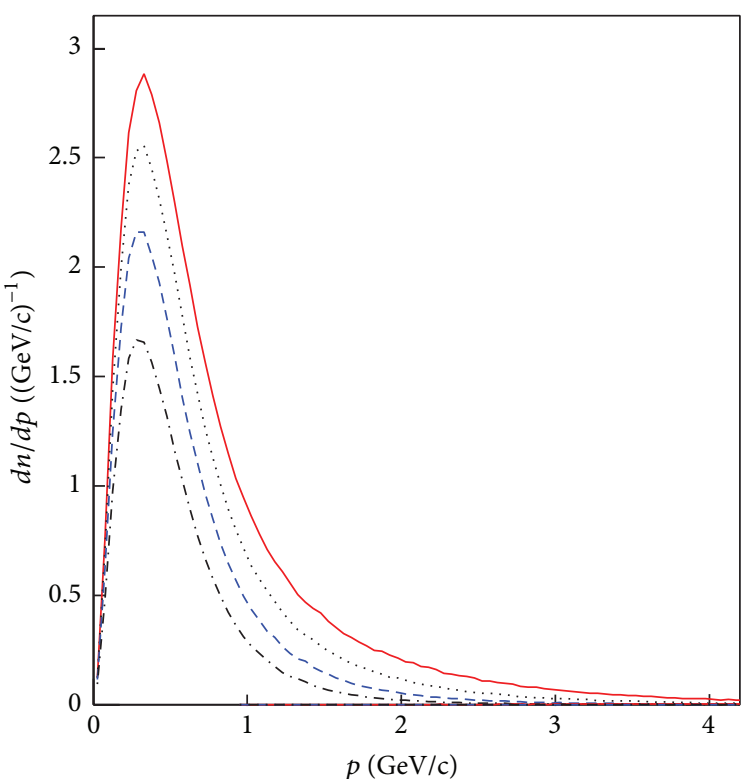

(a)

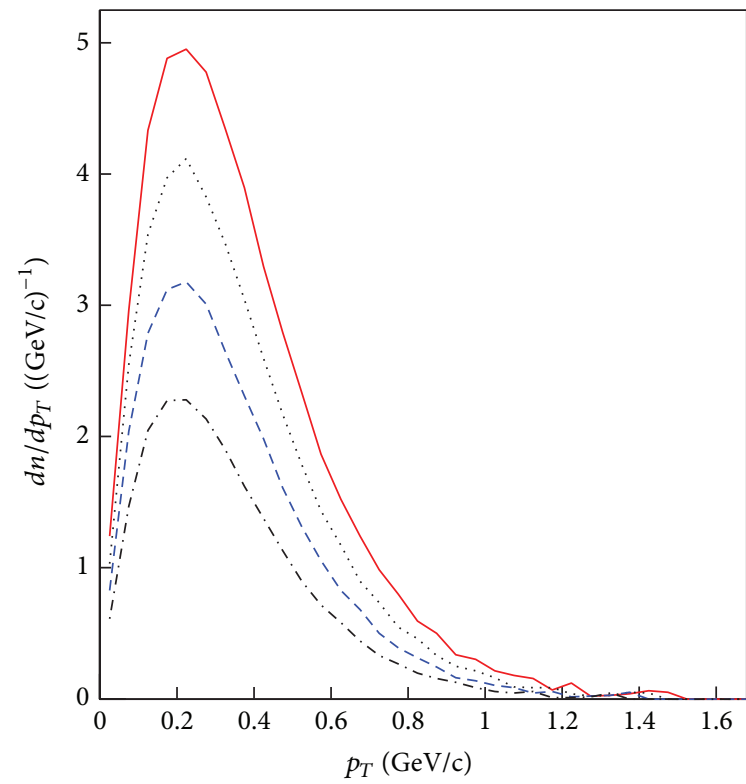

(c)

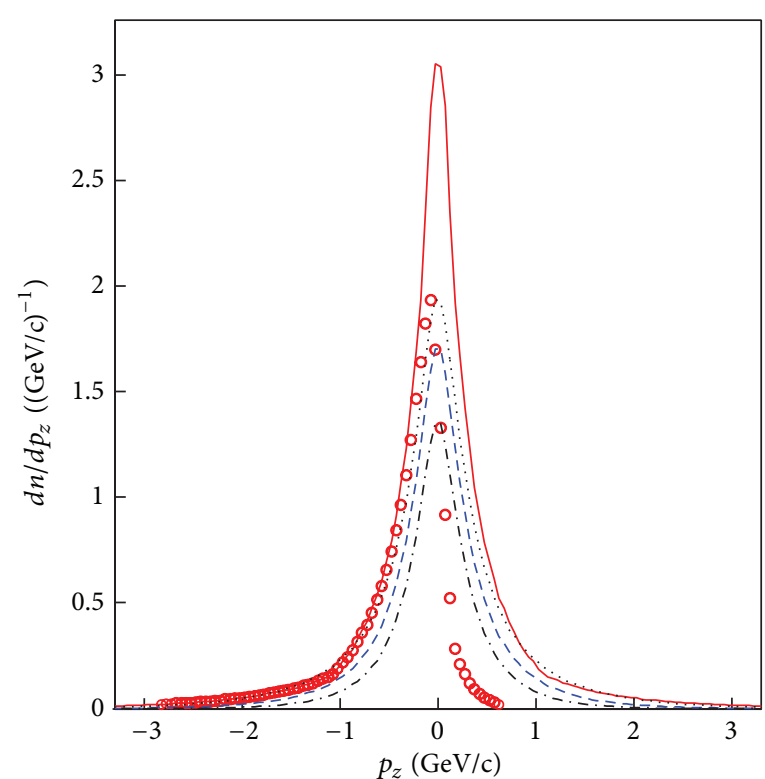

(b)

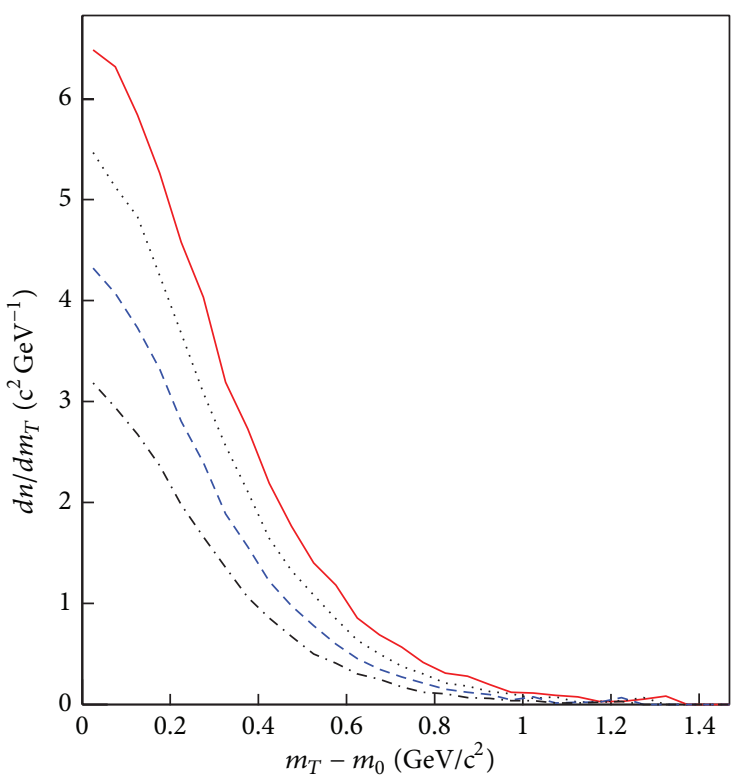

(d)

FIgure 2: Distributions of (a) $p$, (b) $p_{z}$, (c) $p_{T}$, and (d) $m_{T}-m_{0}$ of negatively charged pions produced in $p p$ collisions at the SPS. The solid, dotted, dashed, and dotted-dashed curves correspond to the results at 158, 80, 40, and $20 \mathrm{GeV} / \mathrm{c}$, respectively. The circles in Figure 2(b) represent the contribution of the target cylinder to $p_{z}$ distribution at $158 \mathrm{GeV} / \mathrm{c}$.

a description for the source. Then, we have the momentum $p^{\prime}$ distribution of particles to be

$$
f_{p^{\prime}}\left(p^{\prime}\right)=\frac{1}{n} \frac{d n}{d p^{\prime}}=C p^{\prime 2} \exp \left(-\frac{\sqrt{p^{\prime 2}+m_{0}^{2}}}{T}\right),
$$

where $C=\left(1 / m_{0}^{2} T\right)\left(1 / K_{2}\left(m_{0} / T\right)\right)$ is the normalization constant, $K_{2}\left(m_{0} / T\right)$ is the modified Bessel function of order 2, $m_{0}$ is the rest mass of the considered particle, $n$ is the particle number, and $T$ is the temperature parameter of the source.
The particles are assumed to emit isotropically in the rest frame of the source. Then, the distributions of emission angle $\theta^{\prime}$ and the azimuth $\phi^{\prime}$ can be given by $f_{\theta^{\prime}}\left(\theta^{\prime}\right)=(1 / 2) \sin \theta^{\prime}$ and $f_{\phi^{\prime}}\left(\phi^{\prime}\right)=1 /(2 \pi)$, respectively.

Let $p_{z}^{\prime}, p_{T}^{\prime}, p_{x}^{\prime}, p_{y}^{\prime}, m_{T}^{\prime}, E^{\prime}, y^{\prime}$, and $\eta^{\prime}$ denote, respectively, the longitudinal momentum, transverse momentum, $x$-component of momentum, $y$-component of momentum, transverse mass, energy, rapidity, and pseudorapidity of a considered particle in the rest frame of the considered source. In the Monte Carlo calculation, let $r_{1,2,3}$ denote, respectively, 


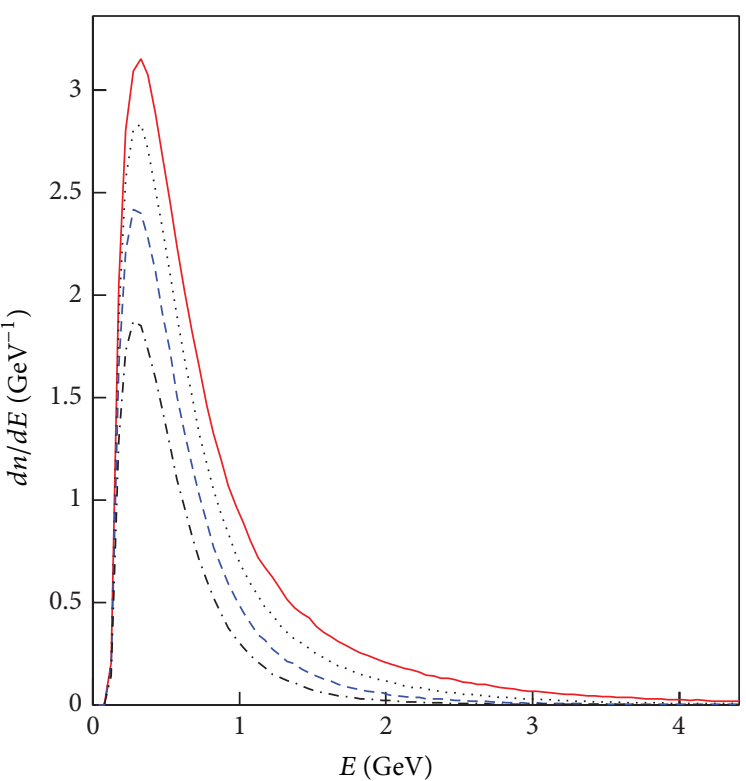

(a)

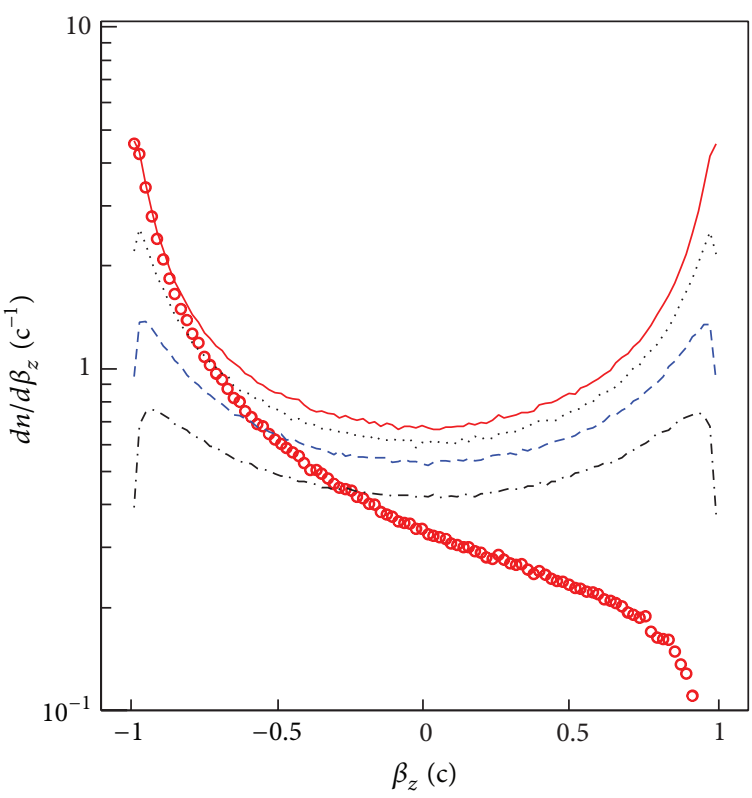

(c)

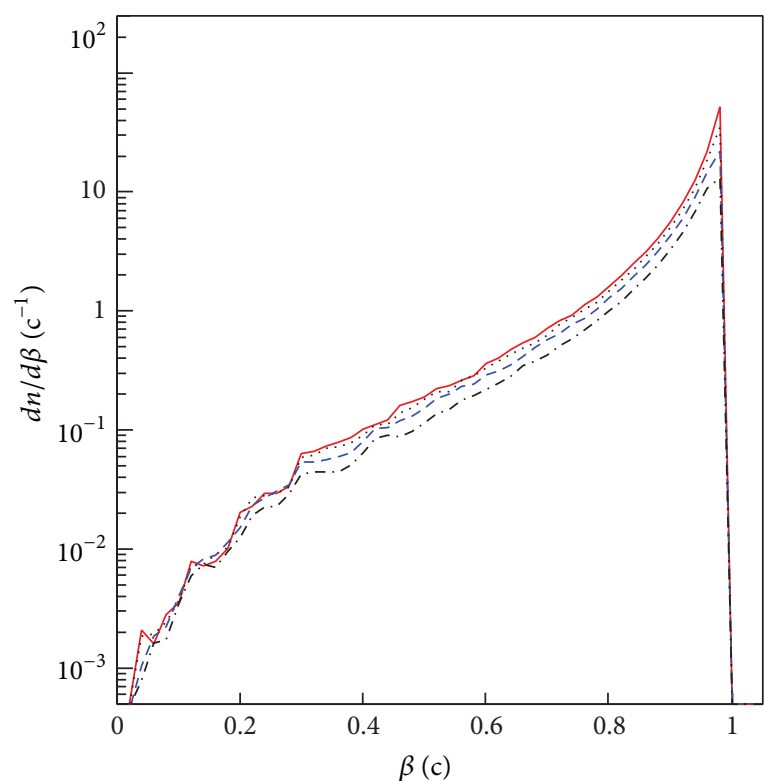

(b)

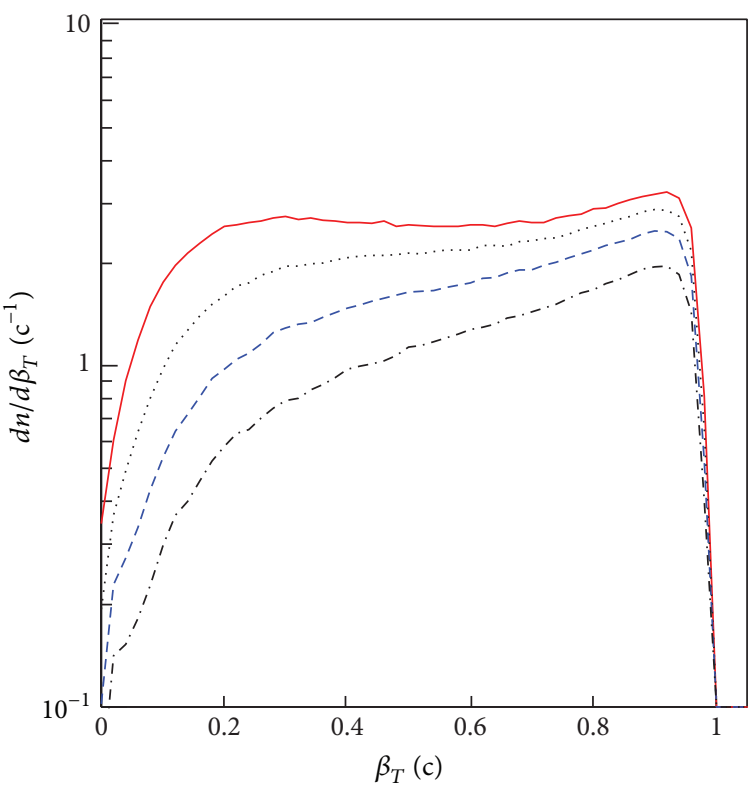

(d)

FIGURE 3: The same as Figure 2, but showing the distributions of (a) $E$, (b) $\beta$, (c) $\beta_{z}$, and (d) $\beta_{T}$. The circles in Figure 3(c) represent the contribution of the target cylinder to $\beta_{z}$ distribution at $158 \mathrm{GeV} / \mathrm{c}$.

random numbers distributed evenly in $[0,1]$. The value of $p^{\prime}$ satisfies

$$
\int_{0}^{p^{\prime}} f_{p^{\prime}}\left(p^{\prime}\right) d p^{\prime}<r_{1}<\int_{0}^{p^{\prime}+d p^{\prime}} f_{p^{\prime}}\left(p^{\prime}\right) d p^{\prime}
$$

For other quantities, we have $\theta^{\prime}=\arctan \left[2 \sqrt{r_{2}\left(1-r_{2}\right)} /\right.$ $\left.\left(1-2 r_{2}\right)\right]$ if the right side of the equation is greater than 0 ; otherwise $\theta^{\prime}=\arctan \left[2 \sqrt{r_{2}\left(1-r_{2}\right)} /\left(1-2 r_{2}\right)\right]+\pi$. Further, we have some quantities such as $\phi^{\prime}=2 \pi r_{3}, p_{z}^{\prime}=p^{\prime} \cos \theta^{\prime}, p_{T}^{\prime}=$ $p^{\prime} \sin \theta^{\prime}, p_{x}^{\prime}=p_{T}^{\prime} \cos \phi^{\prime}, p_{y}^{\prime}=p_{T}^{\prime} \sin \phi^{\prime}, m_{T}^{\prime}=\sqrt{p_{T}^{\prime 2}+m_{0}^{2}}$,
$E^{\prime}=\sqrt{p^{\prime 2}+m_{0}^{2}}=\sqrt{p_{z}^{\prime 2}+p_{T}^{\prime 2}+m_{0}^{2}}=\sqrt{p_{z}^{\prime 2}+m_{T}^{\prime 2}}$, and $y^{\prime} \equiv$ $(1 / 2) \ln \left[\left(E^{\prime}+p_{z}^{\prime}\right) /\left(E^{\prime}-p_{z}^{\prime}\right)\right]$.

In the laboratory or center-of-mass reference frame, let $r_{5,6}$ denote, respectively, random numbers distributed evenly in $[0,1]$; we have the related quantities $y_{x}=\left(y_{T \max }-\right.$ $\left.y_{T \min }\right) r_{5}+y_{T \min }$ if $r_{6}<0.5$, or $y_{x}=\left(y_{P \max }-y_{P \min }\right) r_{5}+y_{P \min }$ if $r_{6}>0.5$. Further, we have $y=y_{x}+y^{\prime}, p_{T}=p_{T}^{\prime}, p_{x}=$ $p_{x}^{\prime}, p_{y}=p_{y}^{\prime}, m_{T}=m_{T}^{\prime}, p_{z}=m_{T} \sinh y, p=\sqrt{p_{T}^{2}+p_{z}^{2}}$, $E=m_{T} \cosh y$, and $\theta=\arctan \left(p_{T} / p_{z}\right)$ if $p_{z}>0$, or $\theta=\arctan \left(p_{T} / p_{z}\right)+\pi$ if $p_{z}<0$. The pseudorapidity $\eta \equiv-\ln \tan (\theta / 2)$. Then, the probability distributions of the 


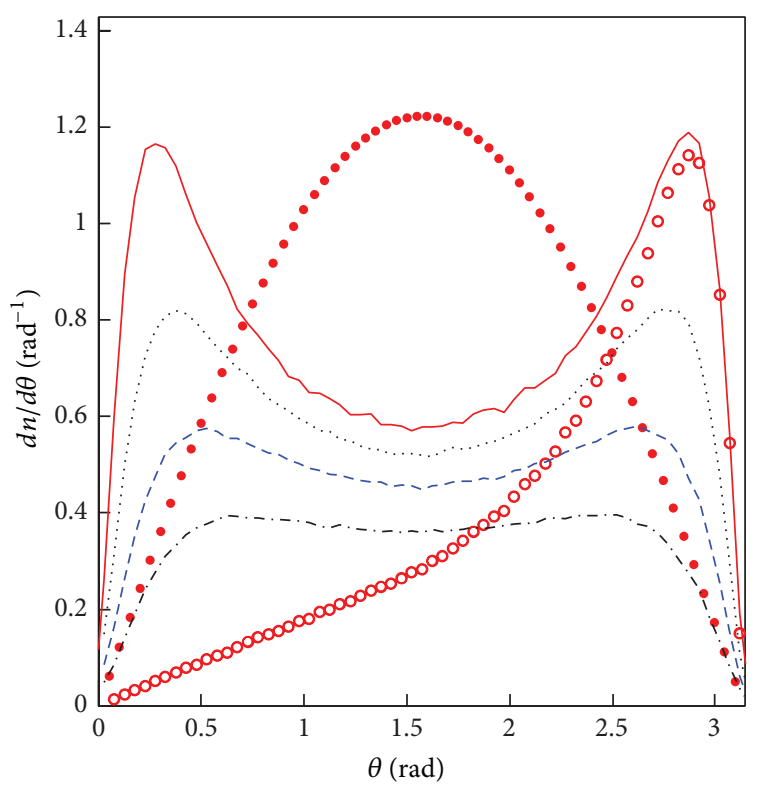

(a)

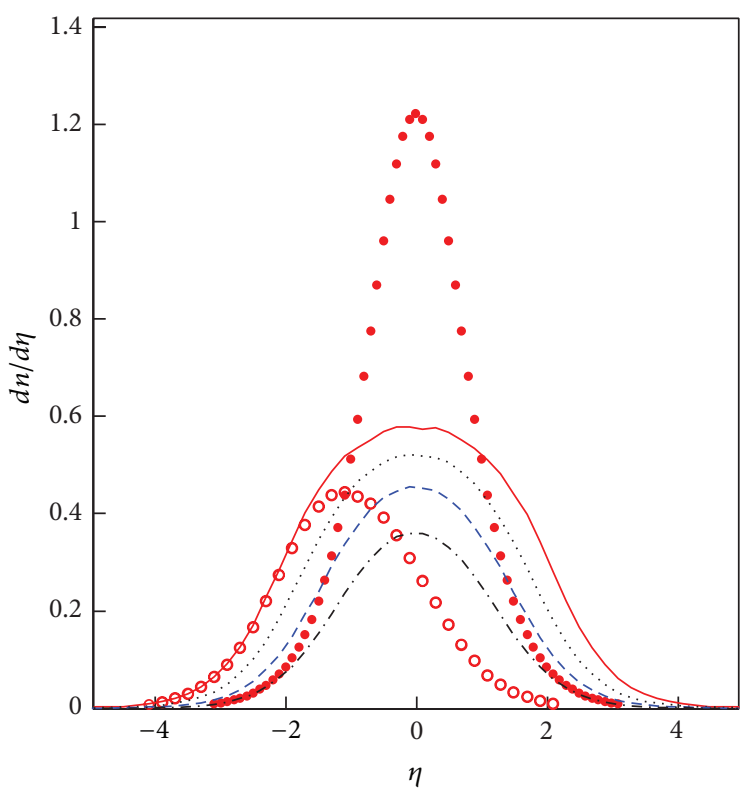

(b)

FIGURE 4: Distributions of (a) $\theta$ and (b) $\eta$ of negatively charged pions produced in $p p$ collisions at the SPS. The solid, dotted, dashed, and dotted-dashed curves correspond to the results at 158,80,40, and $20 \mathrm{GeV} / \mathrm{c}$, respectively. The closed circles show the results of the isotropic emission. The open circles represent the contribution of the target cylinder at $158 \mathrm{GeV} / \mathrm{c}$.

TABLE 1: Values of parameters corresponding to the solid curves in Figure 1.

\begin{tabular}{lccc}
\hline Figure & $T(\mathrm{GeV})$ & $y_{P \max }$ & $y_{P \min }$ \\
\hline Figure 1(a) & $0.150 \pm 0.012$ & $2.00 \pm 0.02$ & $-0.21 \pm 0.01$ \\
Figure 1(b) & $0.145 \pm 0.012$ & $1.71 \pm 0.02$ & $-0.21 \pm 0.01$ \\
Figure 1(c) & $0.141 \pm 0.011$ & $1.43 \pm 0.01$ & $-0.21 \pm 0.01$ \\
Figure 1(d) & $0.138 \pm 0.011$ & $1.20 \pm 0.01$ & $-0.21 \pm 0.01$ \\
\hline
\end{tabular}

considered quantities and their correlations can be obtained. The space density distributions of $p$ and $p_{T}$ are $\rho(p)=$ $\left[1 /\left(4 \pi p^{2} n\right)\right](d n / d p)$ and $\rho\left(p_{T}\right)=\left[1 /\left(2 \pi p_{T} n\right)\right]\left(d n / d p_{T}\right)$, respectively. Besides, some exchangeable quantities in the above expressions are $p_{z}=p_{z}^{\prime} \cosh y_{x}+E^{\prime} \sinh y_{x}, E=$ $p_{z}^{\prime} \sinh y_{x}+E^{\prime} \cosh y_{x}$, and $y \equiv(1 / 2) \ln \left[\left(E+p_{z}\right) /\left(E-p_{z}\right)\right]$.

Further, the velocity and corresponding longitudinal and transverse components of the particle can be given by $\beta=$ $p / E, \beta_{z}=p_{z} / E$, and $\beta_{T}=p_{T} / E$, respectively. If we assume that the time interval from initial collision to the stage of freeze-out is $t_{0}$, then we have the space coordinates $x=$ $t_{0} \beta_{T} \cos \phi, y=t_{0} \beta_{T} \sin \phi, z=r_{z}=t_{0} \beta_{z}, r_{T}=\sqrt{x^{2}+y^{2}}=$ $t_{0} \beta_{T}$, and $r=t_{0} \beta$. Thus, the probability distributions of the considered quantities and their correlations can be obtained. The space density distributions of $r$ and $r_{T}$ are $\rho(r)=$ $\left[1 /\left(4 \pi r^{2} n\right)\right](d n / d r)$ and $\rho\left(r_{T}\right)=\left[1 /\left(2 \pi r_{T} n\right)\right]\left(d n / d r_{T}\right)$, respectively.

\section{Comparison and Extraction}

The NA61/SHINE Collaboration has measured the rapidity distributions and other features of negatively charged pions produced in $20-158 \mathrm{GeV} / \mathrm{c} p p$ collisions at the SPS [1]. It is shown that the rapidity spectrums are parameterized by the sum of two Gaussian functions symmetrically displaced with respect to midrapidity:

$\frac{d n}{d y}$

$$
=\frac{n}{2 \sigma_{0} \sqrt{2 \pi}} \cdot\left[\exp \left(-\frac{\left(y-y_{0}\right)^{2}}{2 \sigma_{0}^{2}}\right)+\exp \left(-\frac{\left(y+y_{0}\right)^{2}}{2 \sigma_{0}^{2}}\right)\right],
$$

where $n, \sigma_{0}$, and $y_{0}$ are the normalization constant, distribution width for one Gaussian, and peak position parameter, respectively. The values with errors of the three parameters at different momenta are obtained by the NA61/SHINE Collaboration [1].

We can compare directly our modelling results with the experimental distribution function of the NA61/SHINE Collaboration [1]. In Figure 1, the rapidity and pseudorapidity distributions of negatively charged pions produced in (a) 158 , (b) 80 , (c) 40 , and (d) $20 \mathrm{GeV} / \mathrm{c} p p$ collisions are displayed. The two-dotted curves represent the rapidity distribution range of the NA61/SHINE experiment [1]. The solid curves which fall into the experimental distribution range are our modelling results obtained by using the multisource thermal model. The modelling results in fact give almost whole superpositions to the two-Gaussian functions which are not presented in the panels. Correspondingly, the modelling results on the pseudorapidity distributions are given by the dashed curves. In the calculation, we have used the Monte Carlo technique and $\chi^{2}$-testing method. The values 


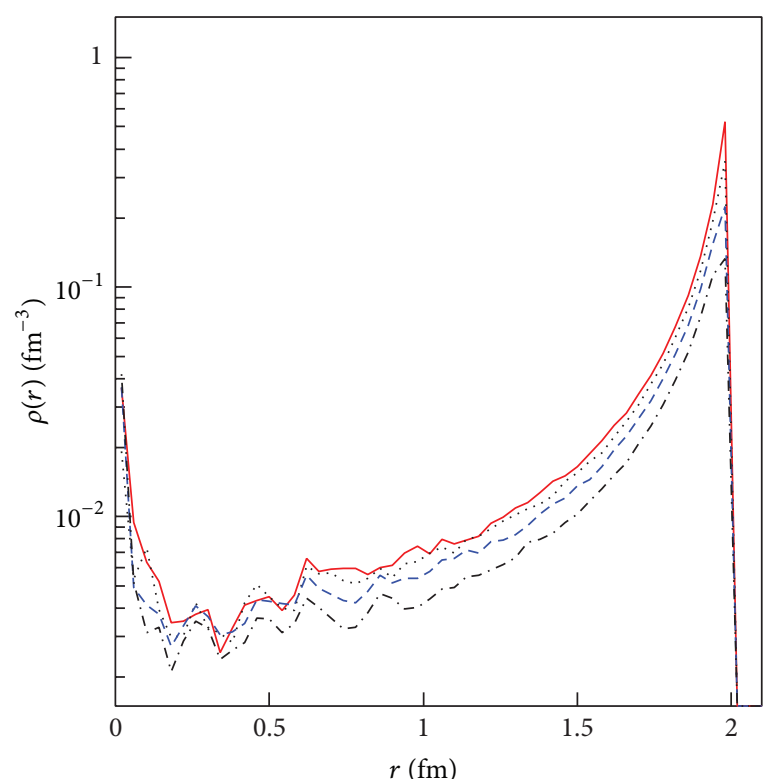

(a)

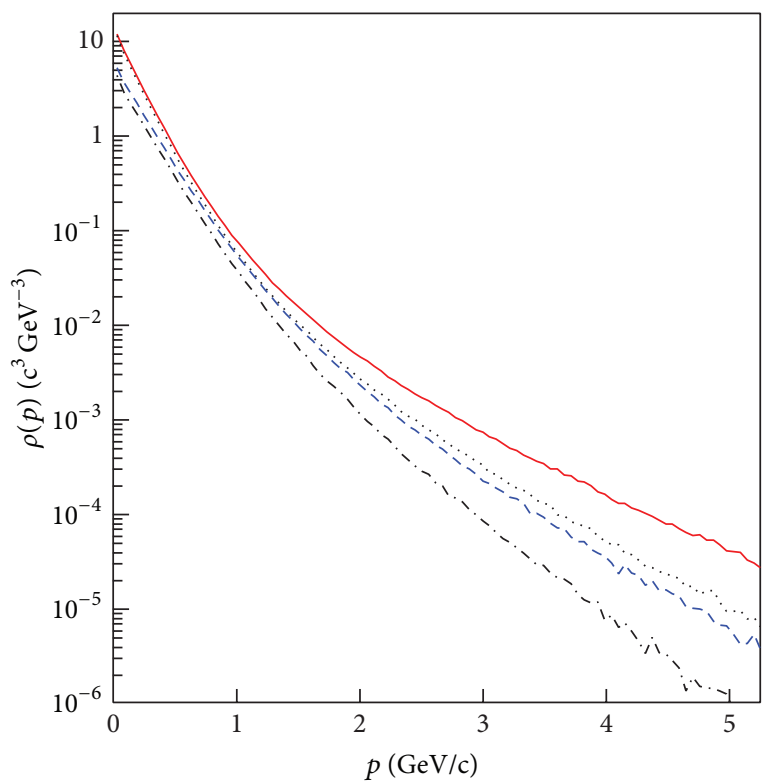

(c)

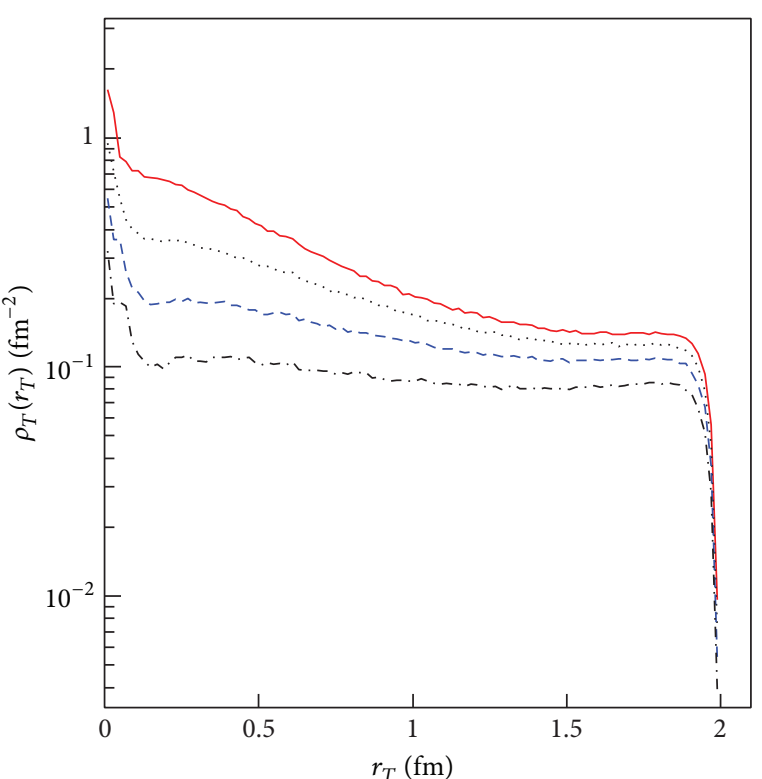

(b)

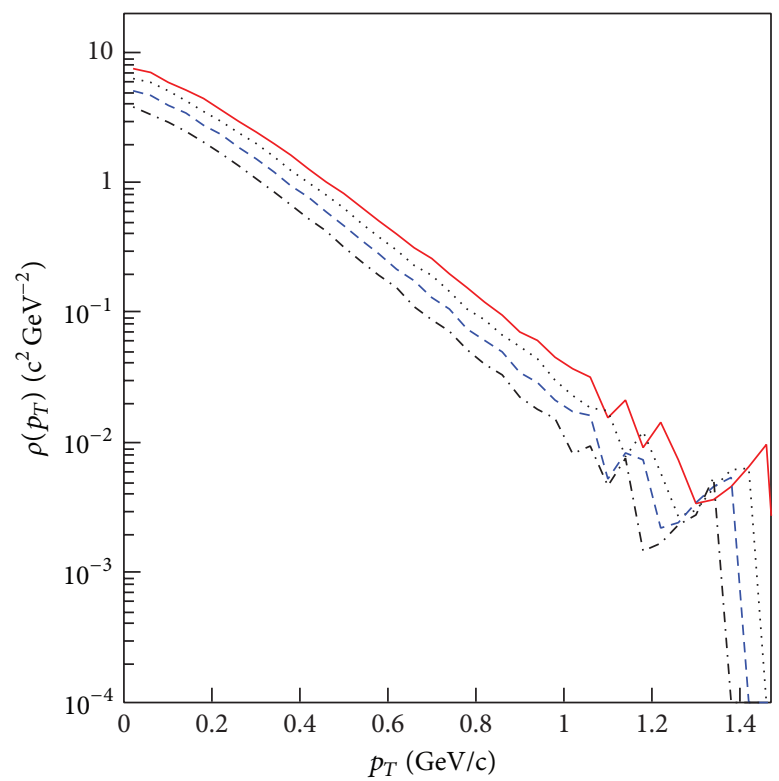

(d)

FIGURE 5: Space density distributions of (a) $r$, (b) $r_{T}$, (c) $p$, and (d) $p_{T}$ of negatively charged pions produced in $p p$ collisions at the SPS. The solid, dotted, dashed, and dotted-dashed curves correspond to the results at $158,80,40$, and $20 \mathrm{GeV} / \mathrm{c}$, respectively.

of parameters $T, y_{P \max }\left(=-y_{T \text { min }}\right)$, and $y_{P \min }\left(=-y_{T \max }\right)$ for Figures 1(a), 1(b), 1(c), and 1(d) are listed in Table 1. One can see that the model describes the experimental rapidity distributions of negatively charged pions produced in $p p$ collisions at the SPS. The pseudorapidity distribution has a lower peak and wider range than those of the rapidity distribution. The temperature increases and the rapidity shift $y_{P \max }$ increases with increase of the incident momentum, and the rapidity shift $y_{P \text { min }}$ does not change with the momentum.

By using the above parameter values, we can obtain some interesting features for other quantities. Figure 2 presents the distributions of (a) $p$, (b) $p_{z}$, (c) $p_{T}$, and (d) $m_{T}-m_{0}$ of negatively charged pions produced in $p p$ collisions at the SPS. The solid, dotted, dashed, and dotted-dashed curves correspond to the results at $158,80,40$, and $20 \mathrm{GeV} / \mathrm{c}$, respectively. The circles in Figure 2(b) represent the contribution of the target cylinder to $p_{z}$ distribution at $158 \mathrm{GeV} / \mathrm{c}$, while the contribution of the projectile cylinder is symmetrical. For the $p, p_{T}$, and $m_{T}-m_{0}$ distributions, both the contributions of the target and projectile cylinders are the same and equal to a half of the distributions. Similarly, Figure 3 presents the distributions of (a) $E$, (b) $\beta$, (c) $\beta_{z}$, and (d) $\beta_{T}$ of the 


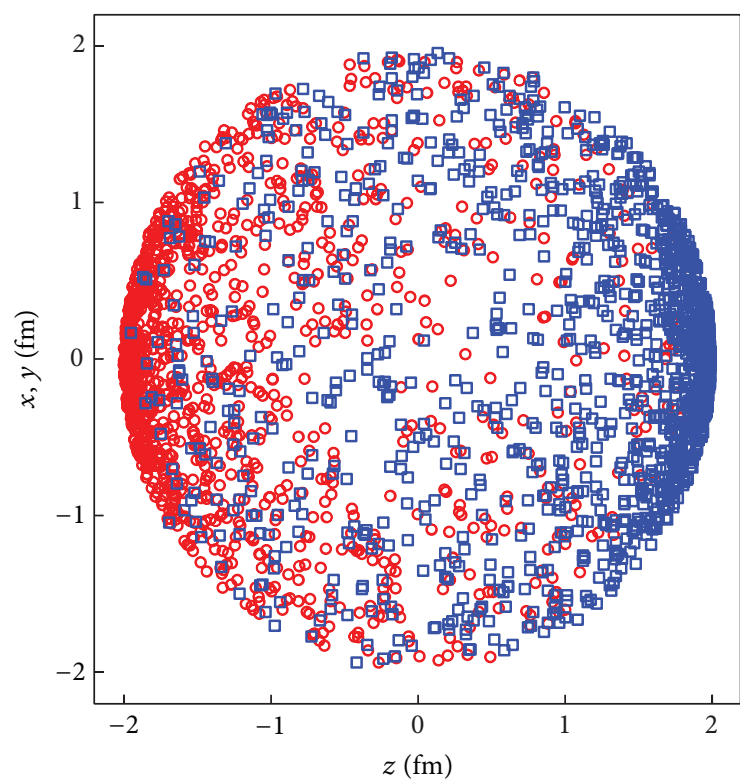

(a)

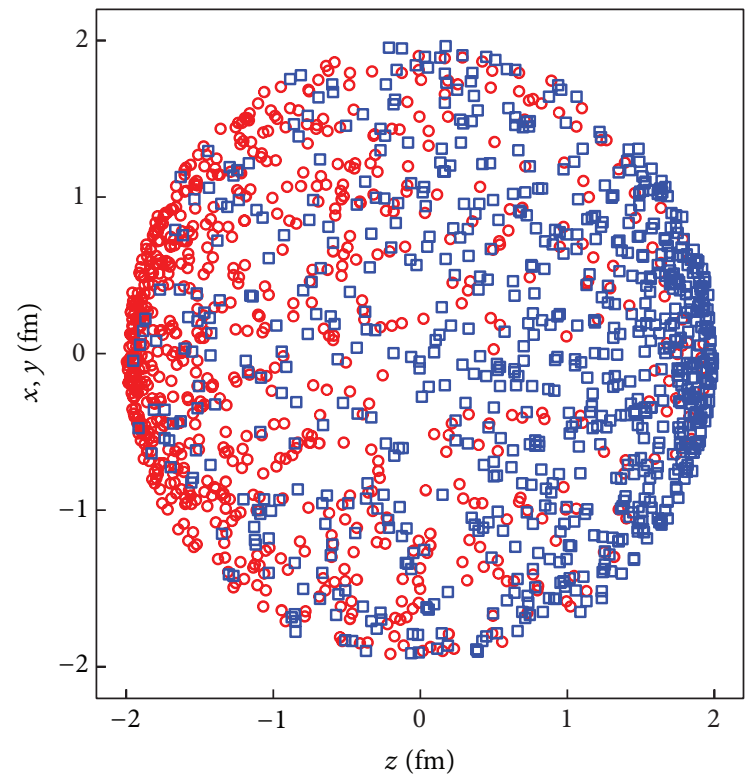

(c)

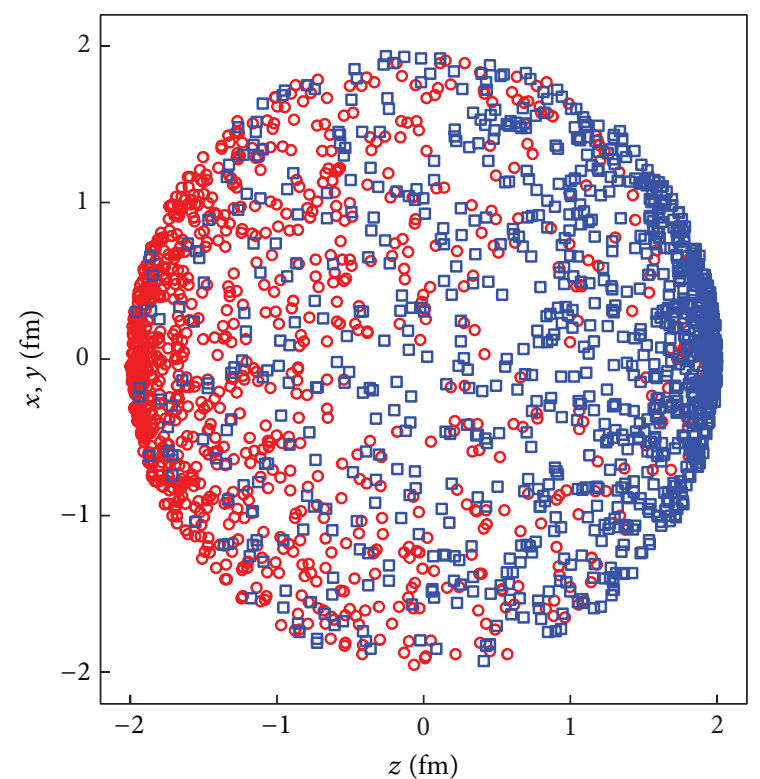

(b)

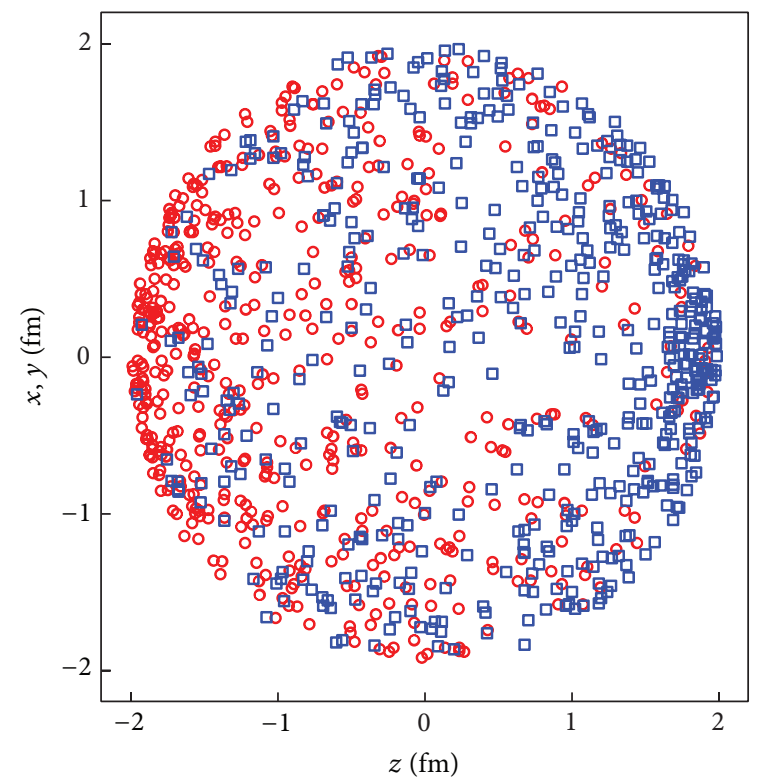

(d)

FIGURE 6: Dispersion plots in $x(y)$ - $z$ planes for negatively charged pions produced in (a) 158, (b) 80 , (c), 40, and (d) $20 \mathrm{GeV} / \mathrm{c} p p$ collisions. The circles and squares correspond, respectively, to the contributions of the target and projectile cylinders in 500 events.

negatively charged pions. The circles in Figure 3(c) represent the contribution of the target cylinder to $\beta_{z}$ distribution at $158 \mathrm{GeV} / \mathrm{c}$, while the contribution of the projectile cylinder is symmetrical. For the $E, \beta$, and $\beta_{T}$ distributions, both the contributions of the target and projectile cylinders are the same and equal to a half of the distributions.

The space angular distributions of negatively charged pions produced in $P p$ collisions at the SPS are displayed in Figure 4(a). The solid, dotted, dashed, and dotted-dashed curves represent the results at $158,80,40$, and $20 \mathrm{GeV} / \mathrm{c}$, respectively. The open circles are the contribution of the target cylinder at $158 \mathrm{GeV} / \mathrm{c}$, while the contribution of the projectile cylinder is symmetrical. For comparison, the result of an isotropic emission is given by the closed circles. To compare the pseudorapidity distributions at different incident momenta, the dashed curves in Figures 1(a)-1(d) are plotted together in Figure 4(b) by the solid, dotted, dashed, and dotted-dashed curves, respectively. The contribution of the target cylinder at $158 \mathrm{GeV} / \mathrm{c}$ is shown by the open circles, while the contribution of the projectile cylinder is symmetrical. For comparison, the result of an isotropic emission is given by the closed circles. One can see that the final-state particles are far from the isotropic emission. 


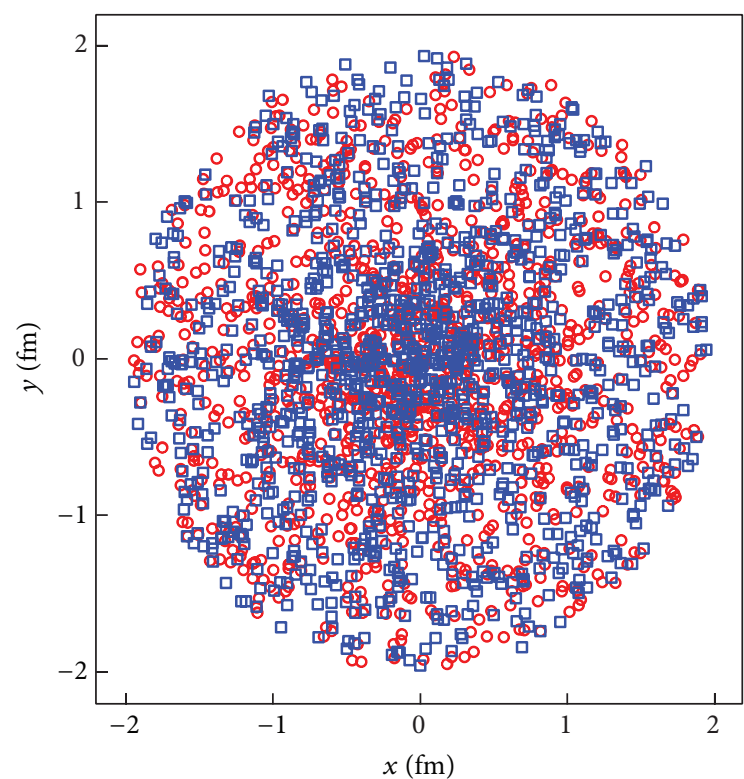

(a)

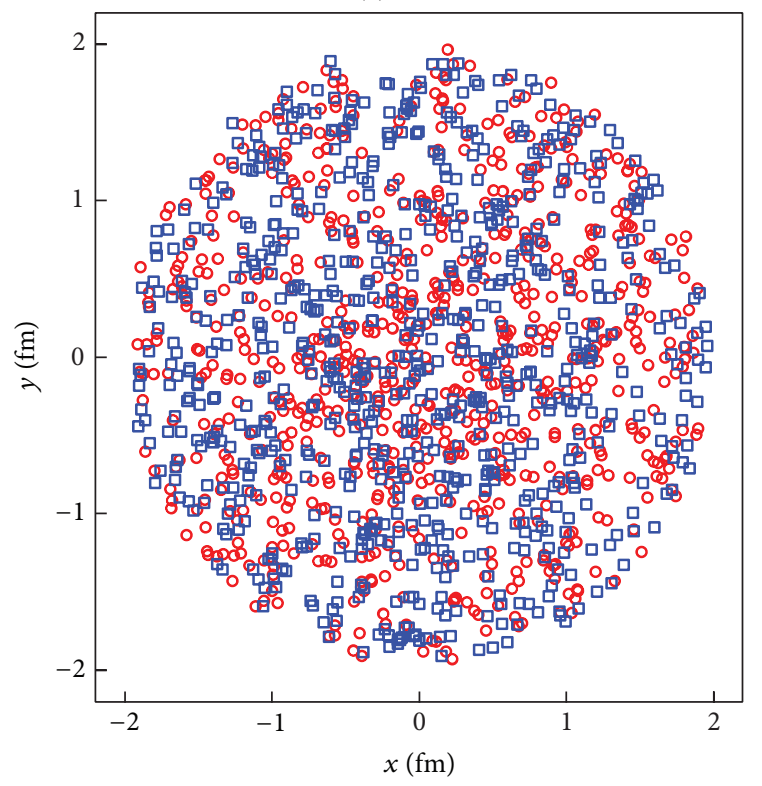

(c)

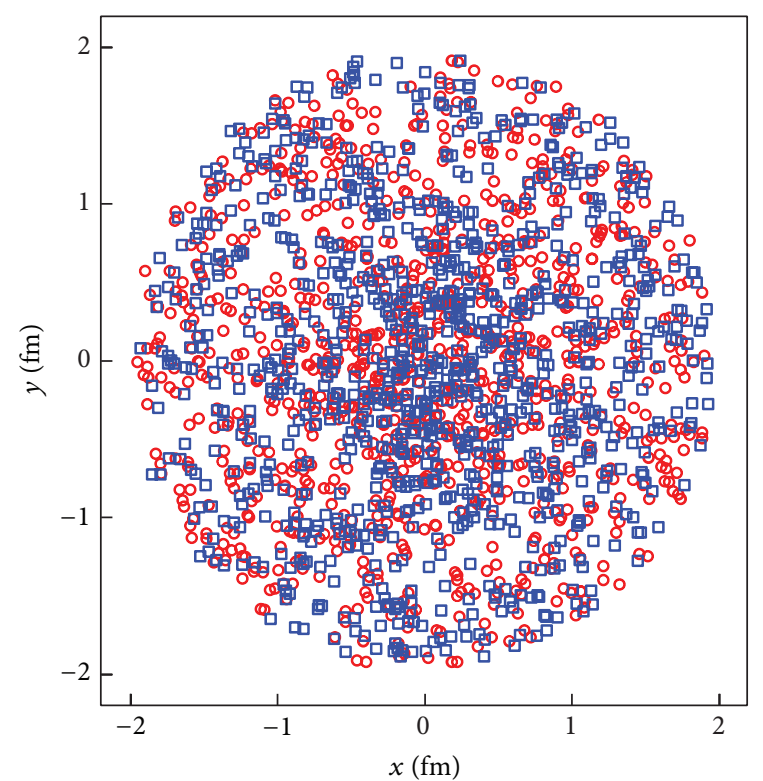

(b)

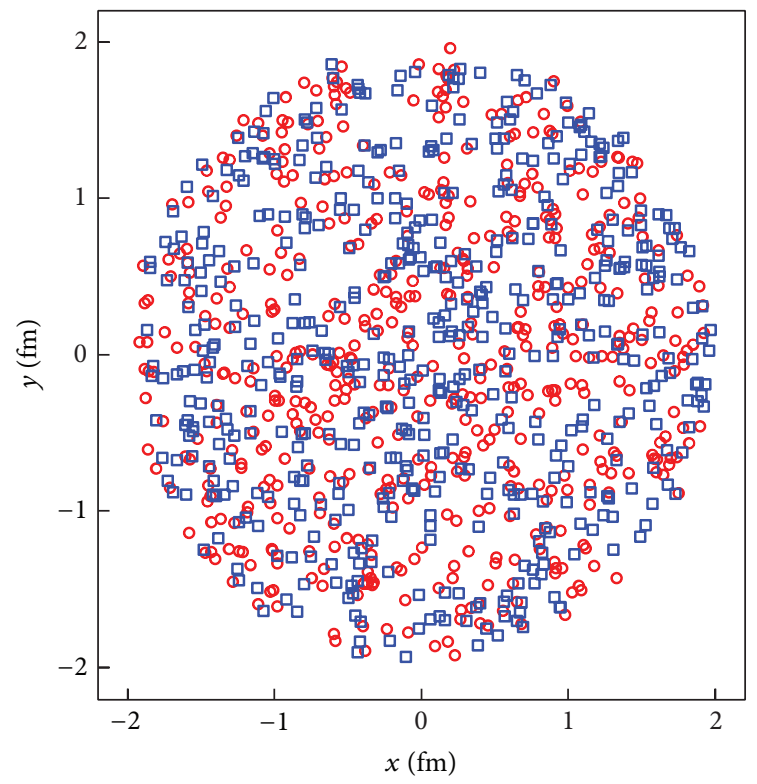

(d)

FIgURE 7: The same as Figure 6, but showing the dispersion plots in $y$ - $x$ planes.

The space density distributions of (a) $r$, (b) $r_{T}$, (c) $p$, and (d) $p_{T}$ are presented in Figure 5, where the value of $t_{0}$ is taken to be $2 \mathrm{fm} / \mathrm{c}$ for convenience. The solid, dotted, dashed, and dotted-dashed curves correspond to the results at $158,80,40$, and $20 \mathrm{GeV} / \mathrm{c}$, respectively. One can see that the maximum $\rho(r)$ appears at the maximum $r$ and the minimum $\rho(r)$ appears in the region close to 0 . The maximum values of $\rho\left(r_{T}\right), \rho(p)$, and $\rho\left(p_{T}\right)$ appear at 0 and the minimum values appear at the maximum coordinate or momentum (transverse momentum) values.

The dispersion plots in $x(y)-z, y-x$, and $p_{x}\left(p_{y}\right)-p_{z}$ planes for negatively charged pions produced in (a) 158 , (b) 80 , (c), 40 , and (d) $20 \mathrm{GeV} / \mathrm{c} p p$ collisions are presented in Figures 6,7 , and 8 , respectively. The circles and squares correspond, respectively, to the contributions of the target and projectile cylinders in 500 events. We can see that the density in large $|z|$ and small $|x(y)|$ region is greater than those in other regions. The density in small $\left|p_{x}\left(p_{y}\right)\right|$ and $\left|p_{z}\right|$ region is greater than those in other regions. The contribution points of the two cylinders are mixed in small longitudinal momentum region. The particle number densities in coordinate space and momentum space increase obviously with increase of the incident momentum.

\section{Conclusions}

From the above discussions, we obtain the following conclusions. 


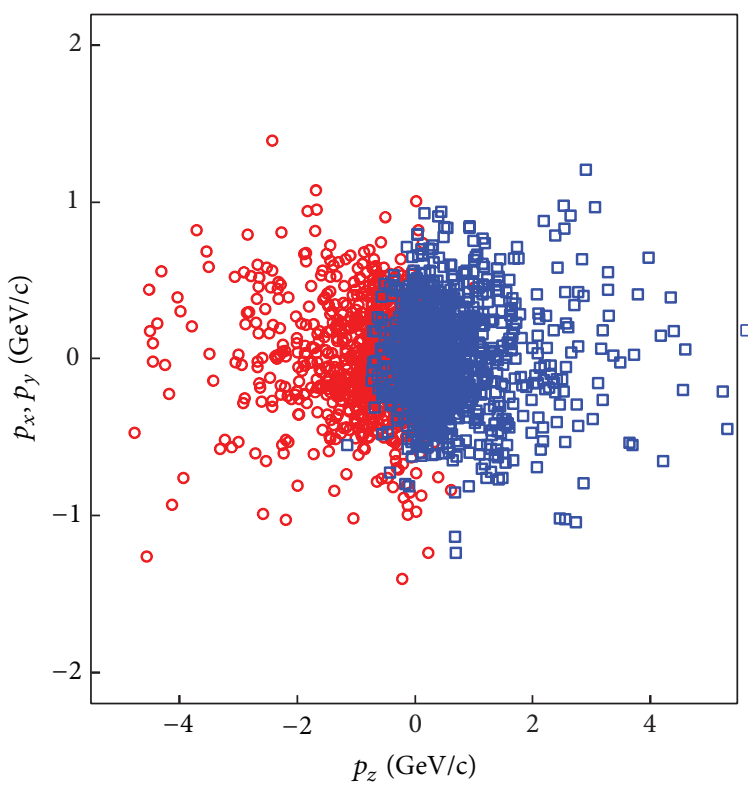

(a)

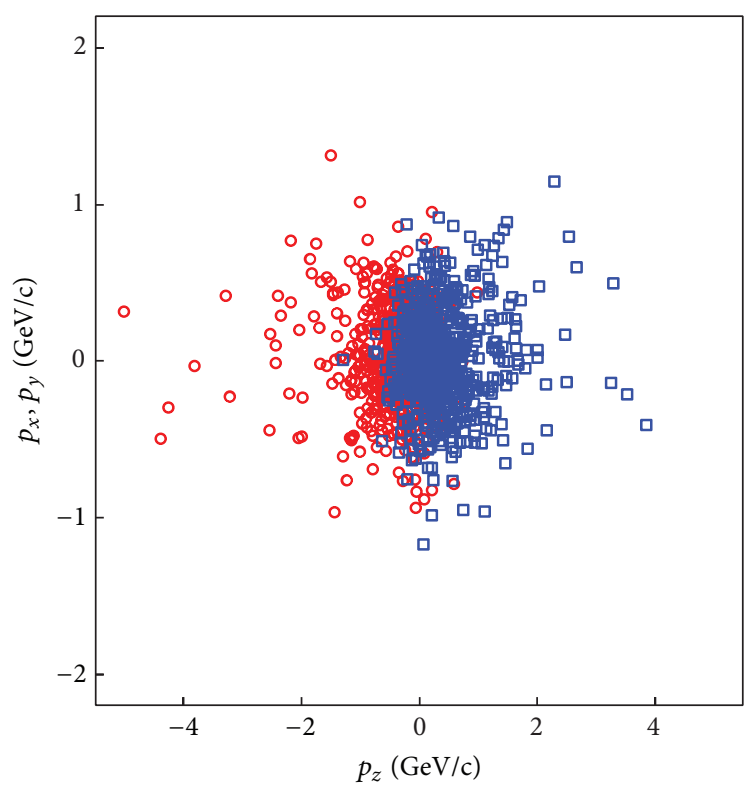

(c)

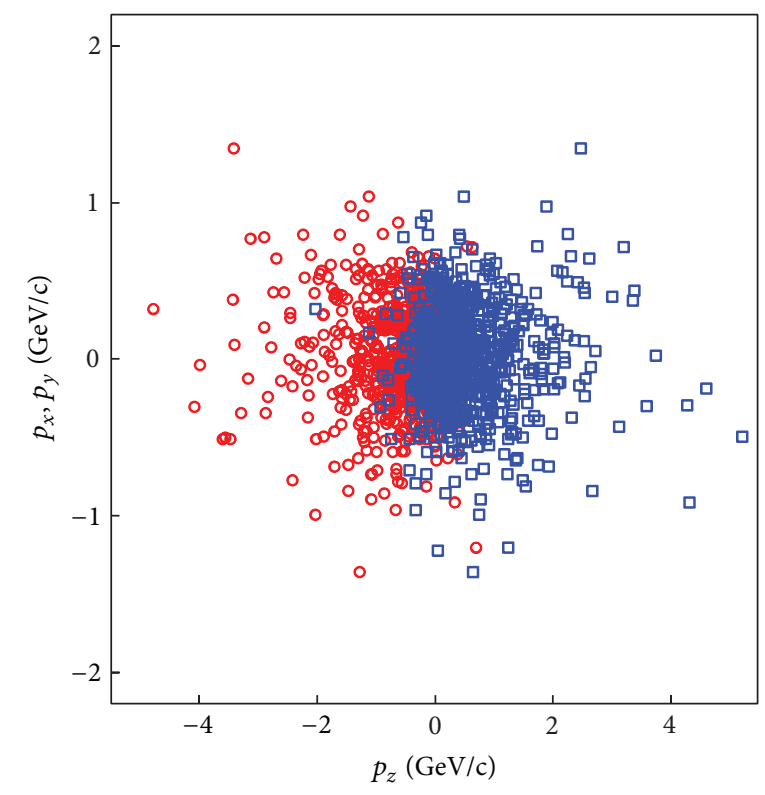

(b)

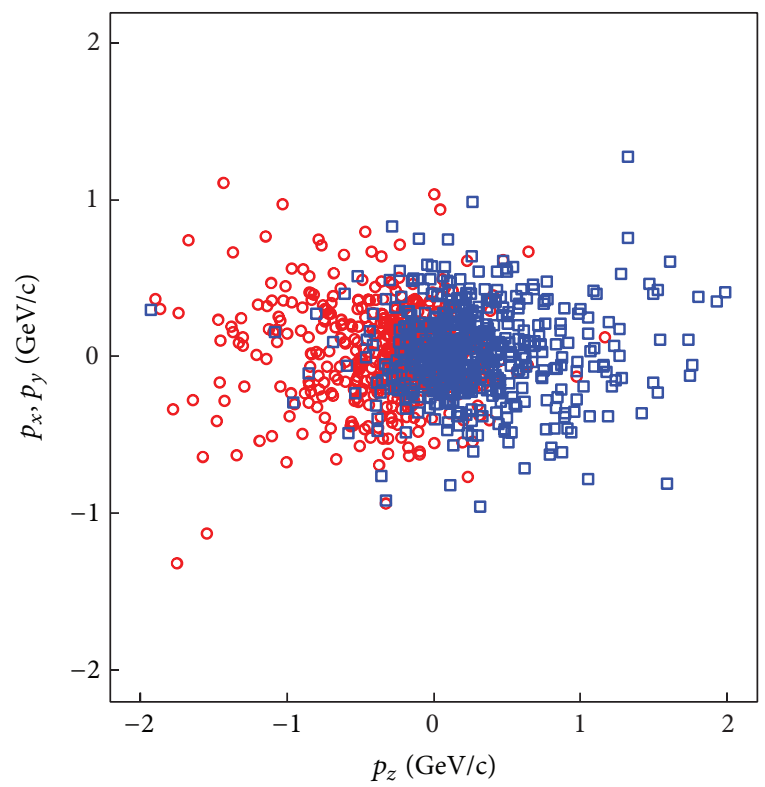

(d)

FIgURE 8: The same as Figure 6, but showing the dispersion plots in $p_{x}\left(p_{y}\right)-p_{z}$ planes.

(a) The multisource thermal model is used to describe the rapidity distributions of negatively charged pions produced in $p p$ collisions at SPS energies (momenta). A target cylinder and a projectile cylinder are assumed to form in the collisions. For each source, a relativistic ideal gas model is used. The calculated results are in agreement with the rapidity distributions measured by the NA61/SHINE Collaboration [1]. In our calculation, the main parameters are the temperature, the maximum rapidity shift, and the minimum rapidity shift. The temperature and the maximum rapidity shift increase with increase of the incident momentum, and the minimum rapidity shift does not change with the momentum. (b) According to the parameter values obtained from the rapidity distributions, the other distributions are obtained in the framework of the multisource thermal model. The other distributions include the probability distributions of momenta, longitudinal momenta, transverse momenta (transverse masses), energies, velocities, longitudinal velocities, transverse velocities, space angles, and pseudorapidities, as well as the number density distributions of particles in coordinate and momentum spaces. The final-state particles are far from the isotropic emission. The maximum $\rho(r)$ appears at the maximum $r$ and the minimum $\rho(r)$ appears in the region close to 0 . The maximum values of $\rho\left(r_{T}\right), \rho(p)$, 
and $\rho\left(p_{T}\right)$ appear at 0 and the minimum values appear at the maximum coordinate or momentum (transverse momentum) values.

(c) According to the parameter values obtained from the rapidity distributions, the particle dispersion plots in $x(y)-z$, $y-x$, and $p_{x}\left(p_{y}\right)-p_{z}$ planes are also obtained in the framework of the model. The density in large $|z|$ and small $|x(y)|$ region is greater than those in other regions, and the density in small $\left|p_{x}\left(p_{y}\right)\right|$ and $\left|p_{z}\right|$ region is greater than those in other regions. The contributions of the target cylinder and the projectile cylinder are mixed in small longitudinal momentum region.

\section{Conflict of Interests}

The authors declare that there is no conflict of interests regarding the publication of this paper.

\section{Acknowledgments}

This work was partly finished at the State University of New York at Stony Brook, USA. One of the authors (Fu-Hu Liu) thanks Professor Dr. Roy A. Lacey and the members of the Nuclear Chemistry Group of Stony Brook University for their hospitality. The authors acknowledge the supports of the National Natural Science Foundation of China (under Grant no. 10975095, no. 11247250, and no. 11005071), the China National Fundamental Fund of Personnel Training (under Grant No. J1103210), the Open Research Subject of the Chinese Academy of Sciences Large-Scale Scientific Facility (under Grant no. 2060205), the Shanxi Scholarship Council of China, and the Overseas Training Project for Teachers at Shanxi University.

\section{References}

[1] N. Abgrall, A. Aduszkiewicz, Y. Ali et al., "Measurement ofnegatively charged pion spectra in inelastic $\mathrm{p}+\mathrm{p}$ interactions at 20 , 31, 40, 80 and $158 \mathrm{GeV} / \mathrm{c}$," submitted, http://arXiv:1310.2417v2.

[2] M. I. Adamovich, M. M. Agarwall, Y. A. Alexandrov et al., "Angular distributions of light projectile fragments in deep inelastic $\mathrm{Pb}+$ Em interactions at $160 \mathrm{~A} \mathrm{GeV}$," The European Physical Journal A, vol. 6, no. 4, pp. 421-425, 1999.

[3] M. M. Aggarwal, Z. Ahammed, A. L. S. Angelis et al., "Event- byevent charged-neutral fluctuations in $\mathrm{Pb}+\mathrm{Pb}$ collisions at $158 \mathrm{~A}$ GeV," Physics Letters B, vol. 701, no. 3, pp. 300-305, 2011.

[4] M. M. Aggarwal, A. L. S. Angelis, V. Antonenko et al., "Multiplicity and pseudorapidity distribution of photons in $\mathrm{S}+\mathrm{Au}$ reaction at 200A GeV," Physical Review C, vol. 58, no. 2, pp. 1146$1154,1998$.

[5] I. G. Bearden, D. Beavis, C. Besliu et al., "Charged particle densities from $\mathrm{Au}+\mathrm{Au}$ collisions at $\sqrt{s_{N N}}=130 \mathrm{GeV}$," Physics Letters B, vol. 523, no. 3-4, pp. 227-233, 2001.

[6] K. Adcox, S. S. Adler, S. Afanasiev et al., "Formation of dense partonic matter in relativistic nucleus-nucleus collisions at RHIC: experimental evaluation by the PHENIX Collaboration," Nuclear Physics A, vol. 757, no. 1-2, pp. 184-283, 2005.

[7] B. B. Back, M. D. Baker, D. S. Barton et al., "Charged-particle pseudorapidity density distributions from $\mathrm{Au}+\mathrm{Au}$ collisions at $\sqrt{s_{N N}}=130 \mathrm{GeV}$," Physical Review Letters, vol. 87, no. 10, Article ID 102303, 4 pages, 2001.
[8] K. H. Ackermann, N. Adams, C. Adler et al., "Elliptic flow in $\mathrm{Au}+\mathrm{Au}$ Collisions at $\mathrm{p} \sqrt{s_{N N}}=130 \mathrm{GeV}$," Physical Review Letters, vol. 86, no. 3, pp. 402-407, 2001.

[9] K. Aamodt, A. Abrahantes Quintana, D. Adamová et al., "Rapidity and transverse momentum dependence of inclusive $J / \psi$ production in pp collisions at $\sqrt{s}=7 \mathrm{TeV}$," Physics Letters $B$, vol. 704, no. 5, pp. 442-455, 2011, Erratum vol. 718, no. 2, pp. 692-698, 2012.

[10] G. Aad, T. Abajyan, B. Abbott et al., "Measurement of Upsilon production in $7 \mathrm{TeV} p p$ collisions at ATLAS," Physical Review D, vol. 87, no. 5, Article ID 052004, 31 pages, 2013.

[11] V. Khachatryan, A. M. Sirunyan, A. Tumasyan, C. M. S. Collaboration et al., "Upsilon production cross section in pp collisions at $\sqrt{s}=7 \mathrm{TeV}$," Physical Review D, vol. 83, no. 11, Article ID 112004, 27 pages, 2011.

[12] R. Aaij, B. Adeva, M. Adinolfi et al., "Measurement of $J / \psi$ production in pp collisions at $\sqrt{s}=7 \mathrm{TeV}$," The European Physical Journal C, vol. 71, no. 5, Article ID 1645, 17 pages, 2011.

[13] R. Singh, L. Kumar, P. K. Netrakanti, and B. Mohanty, "Selected experimental results from heavy-ion collisions at LHC," Advances in High Energy Physics, vol. 2013, Article ID 761474, 22 pages, 2013.

[14] Y.-P. Liu, C. M. Ko, and T. Song, "Hot medium effects on $J / \psi$ production in $\mathrm{p}+\mathrm{Pb}$ collisions at $\mathrm{p} \sqrt{s_{N N}}=5.02 \mathrm{TeV}$," Physics Letters B, vol. 728, pp. 437-442, 2014.

[15] A. K. Chaudhuri, "Viscous hydrodynamic model for relativistic heavy ion collisions," Advances in High Energy Physics, vol. 2013, Article ID 693180, 25 pages, 2013.

[16] S. V. Akkelin and M. Yu. Sinyukov, "Entanglement of scales as a possible mechanism for decoherence and 6 thermalization in relativistic heavy ion collisions," Physical Review C, vol. 89, no. 3, Article ID 034910, 9 pages, 2014.

[17] E. Marji, A. Canul, Q. MacPherson et al., "Nonperturbative renormalization of the chiral nucleon-nucleon interaction up to next-to-next-to-leading order," Physical Review C, vol. 88, no. 5, Article ID 054002, 10 pages, 2013.

[18] A. Tawfik, "On the higher moments of particle multiplicity, chemical freeze-out, and QCD critical endpoint," Advances in High Energy Physics, vol. 2013, Article ID 574871, 22 pages, 2013.

[19] C. B. Chiu and R. C. Hwa, "Ridge and transverse correlation without long-range longitudinal correlation," Advances in High Energy Physics, vol. 2013, Article ID 728365, 11 pages, 2013.

[20] S. Abreu, N. Borghini, S. Jeon et al., "Heavy-ion collisions at the LHC-last call for predictions," Journal of Physics G, vol. 35, no. 5, Article ID 054001, 185 pages, 2008.

[21] F.-H. Liu, "Particle production in Au-Au collisions at RHIC energies," Physics Letters B, vol. 583, no. 1-2, pp. 68-72, 2004.

[22] F.-H. Liu, "Dependence of charged particle pseudorapidity distributions on centrality and energy in $\mathrm{p}(\mathrm{d}) \mathrm{A}$ collisions at high energies," Physical Review C, vol. 78, no. 1, Article ID 014902, 6 pages, 2008.

[23] F.-H. Liu, "Unified description of multiplicity distributions of final-state particles produced in collisions at high energies," Nuclear Physics A, vol. 810, no. 1-4, pp. 159-172, 2008. 

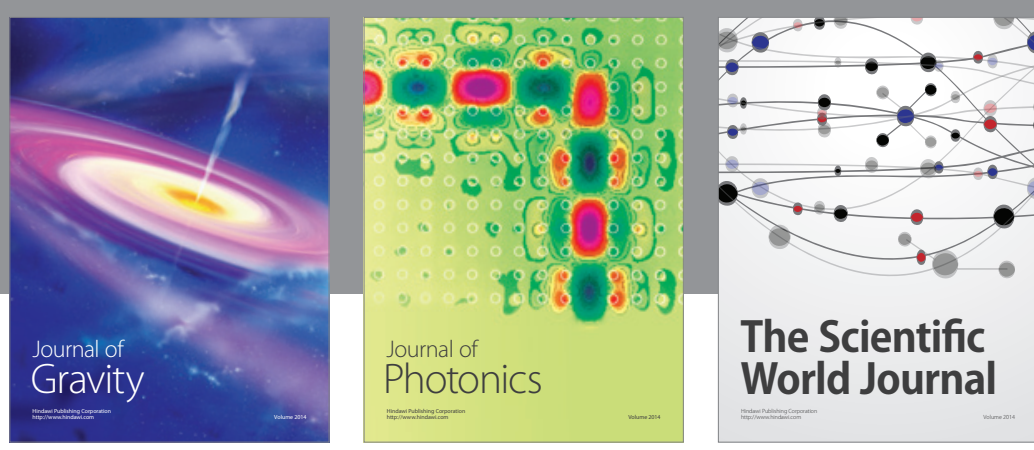

The Scientific World Journal
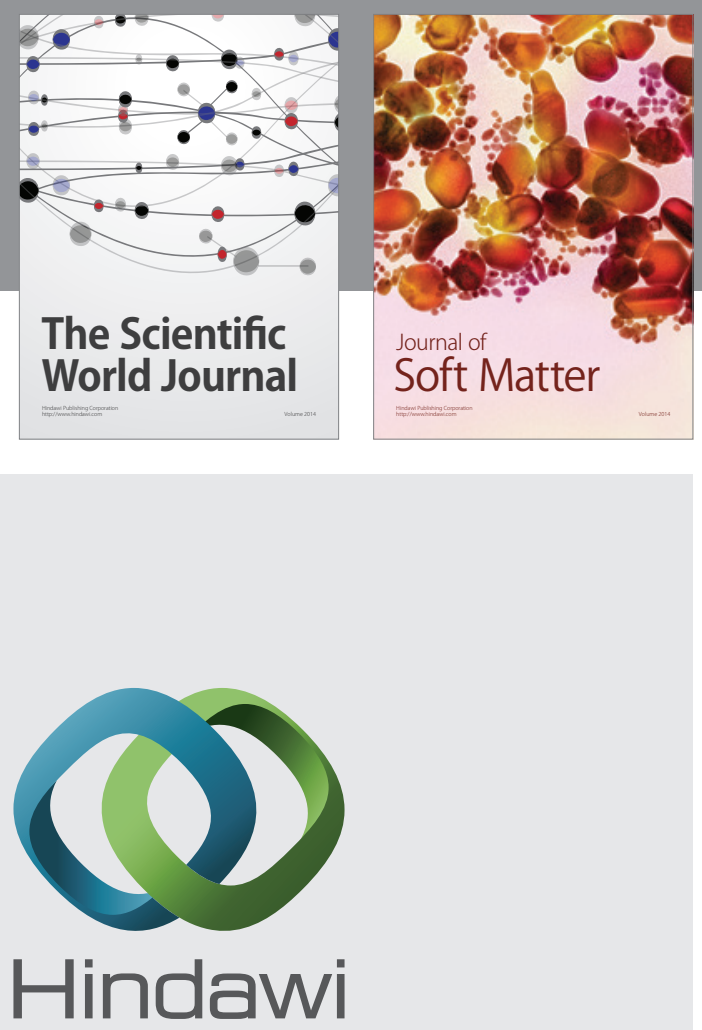

Submit your manuscripts at

http://www.hindawi.com

nternational Journal of

Statistical Mechanics
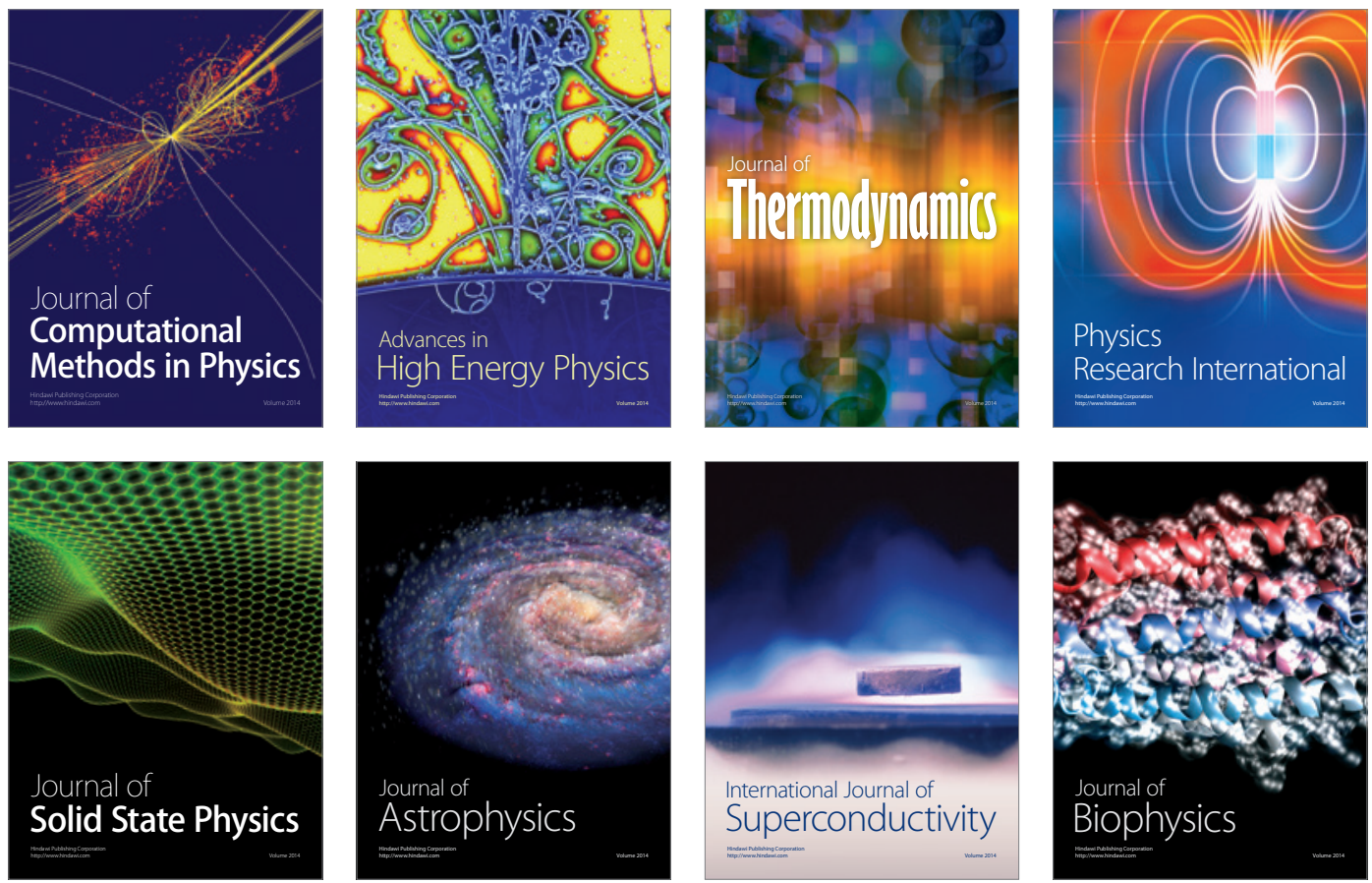
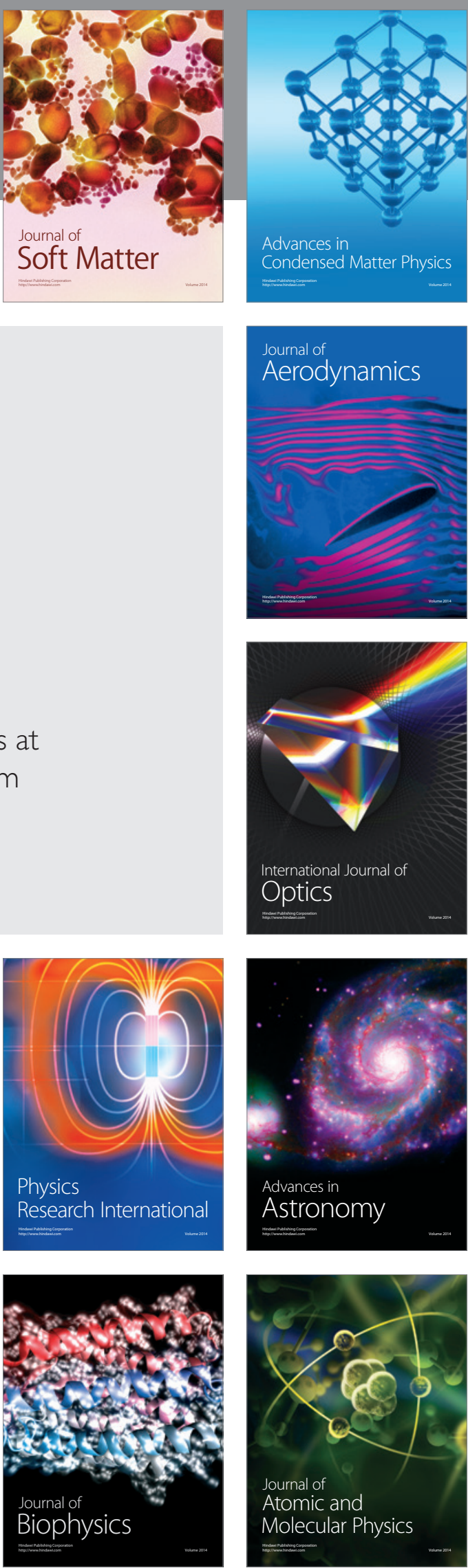